\title{
MAGNETIC DRUG DELIVERY AND TARGETING: PRINCIPLES AND APPLICATIONS
}

\author{
Melania Babincova, Peter Babinec
}

\begin{abstract}
Department of Nuclear Physics and Biophysics, Faculty of Mathematics, Physics, and Informatics, Comenius University, Mlynska dolina F1, 84248 Bratislava, Slovakia

e-mail:babincova@fmph.uniba.sk
\end{abstract}

Received: September 30, 2009; Accepted: November 27, 2009

Key words: Magnetic nanoparticles/Cancer therapy/Drug targeting/Gene Therapy/Controlled drug release

Background: Nanomaterials are at the leading edge of the rapidly developing field of nanotechnology. Magnetic nanoparticles for cancer therapy and diagnosis have been developed on the basis of their unique physico-chemical properties not present in other materials. Their versatility is widely exploited in such diverse techniques as cell and macromolecule separation and purification, immunoassays, targeted drug delivery, controlled material release, electromagnetic hyperthermia, gene therapy, or magnetic resonance imaging. In this review we concentrate on the physical principles of magnetic drug targeting and biomedical applications of this technique.

Methods and results: We examined several databases, PubMed, ISI Web of Knowledge, and Scopus, for the period 1985-2009, with specific attention to studies that used targeting of magnetic nanoparticles especially in the therapy and diagnostics of tumors. We have also presented several of our own results on theoretical simulations of magnetic particle motion in external magnetic field.

Conclusions: We found growing number of published papers in this field of nanomedicine, showing the almost unlimited potential of magnetic nanoparticles in the field of experimental and clinical oncology.

\section{INTRODUCTION}

The history of magnetism dates back to earlier than 600 B.C., but it is only in the twentieth century that scientists have begun to understand it, and develop technologies based on this understanding. Magnetism was most probably first observed in a form of the mineral magnetite called lodestone, which consists of iron oxide a chemical compound of iron and oxygen. The ancient Greeks were the first known to have used this mineral, which they called a magnet (from Greek $\mu \alpha \gamma v \eta \dot{\tau}\rceil \varsigma \lambda i \theta o \varsigma$, "Magnesian stone") because of its ability to attract other pieces of the same material and iron. Curiously, the first systematic study of magnetism using scientific methods was made not by a physicist but by a physician William Gilbert (1540-1603) the most distinguished man of science in England during the reign of Queen Elizabeth I, and the father of electric and magnetic science. Gilbert's principal work is his treatise on magnetism, entitled De magnete, magneticisque corporibus, et de magno magnete tellure (London, 1600). This work, which embodied the results of many years research, was distinguished by its strict adherence to the scientific method of investigation by experiment, and by the originality of its matter, containing, as it does, an account of the author's experiments on magnets and magnetic bodies and on electrical attractions, and also his great conception that the earth is nothing but a large magnet, and that it is this which explains, not only the direction of the magnetic needle north and south, but also the variation and dipping or inclination of the needle. Gilbert's is therefore not merely the first, but the most important, systematic contribution to the sciences of electricity and magnetism.

One of the most promising fields of research lying on the border of medicine, biology, physics, chemistry, and engineering - "nanomedicine", according to the definition given by USA National Institutes of Health is based on the applications of nanotechnology for treatment, diagnosis, monitoring, and control of biological systems. In the forefront of this field is research into the rational delivery and targeting of pharmaceutical, therapeutic, and diagnostic agents. The oldest and still most vivid subfield is applications of magnetic nanoparticles (MNs) in biology and medicine. Multifunctional MNs which are the topic of this review have diverse potential applications in many biological and medical applications such as cell separation, drug targeting, electromagnetic hyperthermia, magnetic resonance contrast enhancement. We began this introduction with the British physician William Gilbert, and we want to close it with an another physician, Vilém Laufberger (1890-1986) from Charles University in Prague who in 1937 isolated for the first time protein ferritin containing $4 \mathrm{~nm}$ large magnetic nanoparticle ${ }^{1}$. In our view the first nanotechnology pioneer should be considered V. Lauberger, although generally is accepted as a beginning of nanotechnology Feynmann visionary lecture "There is a plenty of room at the bottom" delivered in 1959. Ferritin besides its key role in iron body metabolism $^{2}$ has been used for multifold purposes. Let us mention the pathogenesis of Alzheimer ${ }^{3}$ and Parkinson diseases ${ }^{4}$, magnetic cell separation ${ }^{5}$, magnetic drug targeting ${ }^{6}$, MRI contrast agents ${ }^{7}$, electromagnetic hyperthermia ${ }^{8}$, ultrasensitive diagnosis ${ }^{9}$, and macroscopic quantum tunelling ${ }^{10}$. 


\section{SOURCES OF MAGNETIC FIELD}

A magnet is a material or object that produces a magnetic field. This magnetic field is invisible but is responsible for the most notable property of a magnet: a force that pulls on other ferromagnetic materials and attracts or repels other magnets. Permanent magnet materials are widely used in a range of devices from electric and electronic appliances for domestic use to peripheral terminal devices for large-scaled computers. In view of recent needs for miniaturization and high efficiency of electric and electronic equipment, there has been an increasing demand for upgrading of permanent magnet materials. A permanent magnet is one made from a material that stays magnetized. Materials that can be magnetized, which are also the ones that are strongly attracted to a magnet, are called ferromagnetic ${ }^{11}$. These include iron, nickel, cobalt, some rare earth metals and some of their alloys (e.g Alnico), and some naturally occurring minerals such as lodestone. Permanent magnets are made from "hard" ferromagnetic materials which are designed to stay magnetized, while "soft" ferromagnetic materials like soft iron are attracted to a magnet but do not tend to stay magnetized. An electromagnet is made from a coil of wire which acts as a magnet when an electric current passes through it, but stops being a magnet when the current stops. Often an electromagnet is wrapped around a core of ferromagnetic material like steel, which enhances the magnetic field produced by the coil. Because human tissues have a very low level of susceptibility to static magnetic fields, there is no direct scientific evidence showing a health hazard associated with exposure to these fields. However, if a ferromagnetic foreign body is present in human tissue, the magnetic field will interact with it, which can pose a serious safety risk. Specifically, if a pacemaker has been embedded in a patient's chest, care should be taken to keep it away from magnetic fields. It is for this reason that a patient with the device installed cannot be tested with the use of an MRI, which is a magnetic imaging device. The most commonly used ceramic, or ferrite, magnets are made of a sintered composite of powdered iron oxide and barium/strontium carbonate ceramic. Given the low cost of the materials and manufacturing methods, inexpensive magnets (or nonmagnetized ferromagnetic cores, for use in electronic components such as radio antennas, for example) of various shapes can be easily mass-produced. The resulting magnets are noncorroding, but brittle and must be treated like other ceramics. To the another class belongs alnico magnets which are made by casting or sintering a combination of aluminium, nickel and cobalt with iron and small amounts of other elements added to enhance the properties of the magnet. Sintering offers superior mechanical characteristics, whereas casting delivers higher magnetic fields and allows for the design of intricate shapes. Alnico magnets resist corrosion and have physical properties more pliable than ferrite, but not quite as desirable as a metal.

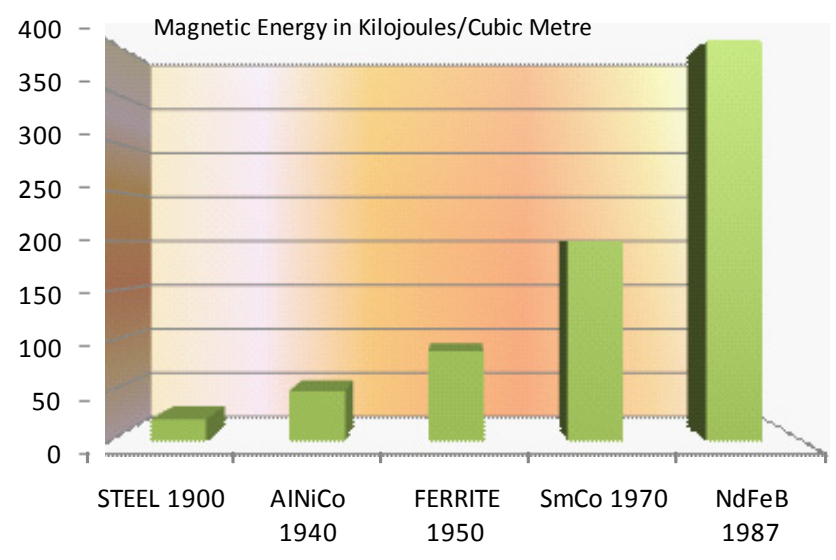

Fig. 1. Comparison of magnetic energy of most commonly used permanent magnets (abbreviations of magnets shown in the figure are explained in the text).

The field of magnetic research has been revolutionarily changed by the introduction of rare-earth magnets are strong, permanent magnets made from alloys of rare earth elements. Rare-earth magnets are substantially stronger than ferrite or alnico magnets. The magnetic field typically produced by rare-earth magnets can be in excess of $120 \mathrm{mT}$, whereas ferrite or ceramic magnets typically exhibit fields of 50 to $100 \mathrm{mT}$. Because rare earth magnets are extremely brittle, they are usually nickel-coated to protect them from breaking and chipping, hence their typically shiny, corrosion resistant nature. Rare earths (lanthanides) are elements with an incompletely filled f-shell. Electrons in such orbitals are strongly localized and therefore easily retain their magnetic moments and function as paramagnetic centers. Magnetic moments in other orbitals are often lost due to strong overlap with the neighbors. In addition, the f-shell can contain up to 7 unpaired electrons, enhancing the size of the magnetic moment. The presence of large magnetic moments is however not sufficient. There also is a strong ferromagnetic coupling between them in these materials. A neodymium magnet is a permanent magnet made of an alloy of neodymium, iron, and boron $-\mathrm{Nd}_{2} \mathrm{Fe}_{14} \mathrm{~B}$. They are the strongest type of permanent magnets made up to now ${ }^{12}$ (Fig. 1). Samarium-cobalt magnets are available since the early 1970s. These samarium cobalt magnet alloys (generally written as $\mathrm{SmCo}_{5}$ ) have one atom of rare earth samarium and five atoms of cobalt. By weight this samarium cobalt magnet alloy will typically contain $36 \%$ samarium with the balance cobalt.

\section{PREPARATION AND FUNCTIONALIZATION OF MAGNETIC NANOPARTICLES AND MICROPARTICLES}

The wet chemical routes to magnetic nanoparticles are simpler, more tractable and more efficient with appreciable control over size, composition and sometimes even the shape of the nanoparticles. Iron oxides (either $\mathrm{Fe}_{3} \mathrm{O}_{4}$ 
or $\mathrm{g}-\mathrm{Fe}_{2} \mathrm{O}_{3}$ ) can be synthesized through the co-precipitation of $\mathrm{Fe}^{2+}$ and $\mathrm{Fe}^{3+}$ aqueous salt solutions by addition of a base ${ }^{13}$. The control of size, shape and composition of nanoparticles depends on the type of salts used (e.g. chlorides, sulphates, nitrates, perchlorates, etc.), $\mathrm{Fe}^{2+}$ and $\mathrm{Fe}^{3+}$ ratio, $\mathrm{pH}$ and ionic strength of the media. Conventionally, magnetite is prepared by adding a base to an aqueous mixture of $\mathrm{Fe}^{2+}$ and $\mathrm{Fe}^{3+}$ chloride at a 1:2 molar ratio. The precipitated magnetite is black in colour. The overall reaction may be written as follows:

$$
\mathrm{Fe}^{2+}+\mathrm{Fe}^{3+}+8 \mathrm{OH}^{-} \rightarrow \mathrm{Fe}_{3} \mathrm{O}_{4}+4 \mathrm{H}_{2} \mathrm{O}
$$

According to the thermodynamics of this reaction, a complete precipitation of $\mathrm{Fe}_{3} \mathrm{O}_{4}$ should be expected between $\mathrm{pH} 9$ and 14, while maintaining a molar ratio of $\mathrm{Fe}^{2+}: \mathrm{Fe}^{3+}$ is $2: 1$ under a non-oxidizing oxygen free environment. Otherwise, $\mathrm{Fe}_{3} \mathrm{O}_{4}$ might also be oxidized as

$$
4 \mathrm{Fe}_{3} \mathrm{O}_{4}+\mathrm{O}_{2}+18 \mathrm{H}_{2} \mathrm{O} \rightarrow 12 \mathrm{Fe}(\mathrm{OH})_{3}
$$

This would critically affect the physical and chemical properties of the nanosized magnetic particles. In order to prevent them from possible oxidation in air as well as from agglomeration, $\mathrm{Fe}_{3} \mathrm{O}_{4}$ nanoparticles produced by these reactions are usually coated with organic or inorganic molecules during the precipitation process. Several authors have reported the magnetic iron oxide nanoparticles coated with silica ${ }^{14}$. An advantage of having a surface enriched in silica is the presence of surface silanol groups that can easily react with alcohols and silane coupling agents ${ }^{15}$. Various biological molecules such as antibodies, proteins, targeting ligands, etc., may also be bound to the polymer surfaces onto the nanoparticles by

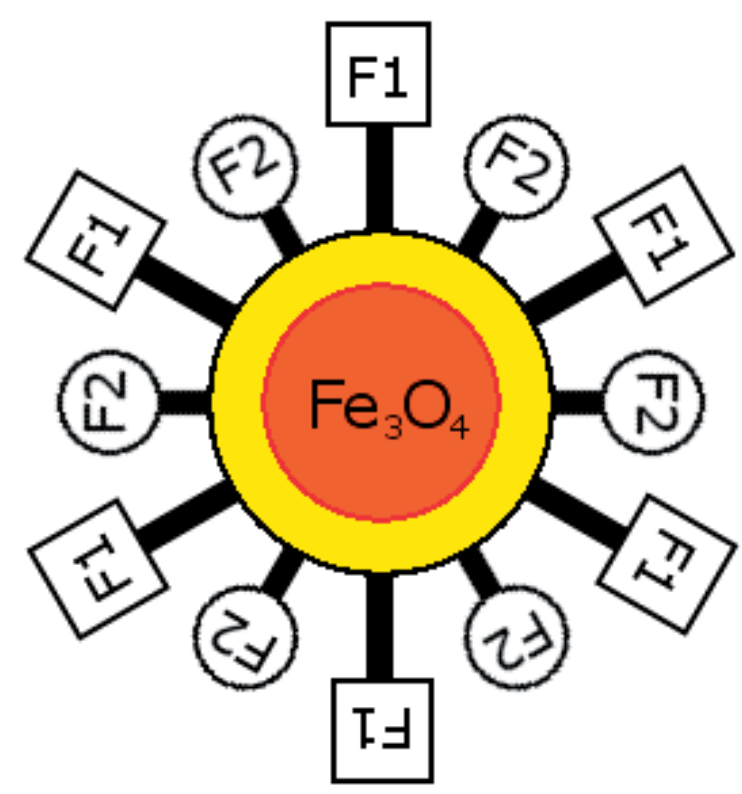

Fig. 2. An example of multifunctionalized (F1 and F2 are examples two different functional groups) magnetic nanoparticle with ferrite core covered by stabilization shell, e.g. $\mathrm{SiO}_{2}$, and conjugated functional groups at the surface. chemically coupling via amide or ester bonds to make the particles target specific (Fig. 2). The possibilities of targeting protein coatings are numerous ${ }^{16-21}$. Another possibility is represented by liposomes ${ }^{22-26}$ with the ability to encapsulate a large number of magnetic nanoparticles and deliver them together, avoiding dilution, to a target site. Combining a therapeutic agent in the payload further enhances the multifunctionality of these delivery vehicles.

\section{MAGNETIC DRUG TARGETING}

The major problems of chemotherapeutics come essentially from the relative lack of specificity derived from their systemic biodistribution and the subsequent side effects provoked by the drug attacking both healthy and target cells ${ }^{27}$.

There are several forces acting on magnetic particles in viscous environment and magnetic field, such as magnetic force due to all field sources, Stokes' viscous drag force, inertia, buoyancy and gravity, thermal kinetics (Brownian motion), and of course particle fluid interactions and interparticle effects like magnetic dipole interactions, electric double layer interactions, and van der Vaal? Walls force. Apart from magnetic and viscous drag force there are the others interactions negligible for magnetic microor nanoparticles ${ }^{28,29}$. For this reason we assume in this paper only these two effects. Given the considerations mentioned before, we can predict the trajectory of motion of magnetic particle in magnetic field and viscous fluid ambient using Newton's law:

$$
m_{p} \frac{\mathrm{d} \mathbf{v}_{p}}{\mathrm{~d} t}=\mathbf{F}_{m}+\mathbf{F}_{s},
$$

where $m_{p}$ and $\mathbf{v}_{p}$ are the mass and velocity of the particle, and $\mathbf{F}_{m}$ and $\mathbf{F}_{s}$ are the magnetic and Stoke's drag forces, respectively.

Magnetic force acting on a magnetic particle is determined by using the method of "effective" dipole moment, in which a magnetic particle is replaced by "equivalent" point dipole moment $\mathbf{m}_{p \text {,eff }}$ localized at the center of particle. According to this method, force acting on the dipole is given by

$$
\mathbf{F}_{m}=\mu\left(\mathbf{m}_{p, e f f} \cdot \nabla\right) \mathbf{H}_{a},
$$

where $\mu$ is permeability of fluid ambient, $\mathbf{m}_{p, \text { eff }}$ is "effective" dipole moment of the particle, which depends on externally applied magnetic field intensity $\mathbf{H}_{a}$ at the center of particle, where the dipole moment is localized. A magnetic force is therefore a function of external magnetic field gradient and the magnetization of the particle. Below the saturation the particles are linearly magnetized with their magnitude of magnetic moment increasing in the direction of external field. Beyond the saturation point, magnetic moment magnitude tends to a constant value. According to this magnetization model of particles based on self demagnetization and magnetic saturation developed by Furlani group ${ }^{30}$ the effective dipole moment can be expressed as 


$$
\mathbf{m}_{p, e f f}=V_{p} f\left(H_{a}\right) \mathbf{H}_{a},
$$

we consider magnetic particle with radius $R_{p}$ and volume $V_{p}$, and a function

$$
f\left(H_{a}\right)=\left\{\begin{array}{cl}
\frac{3\left(\chi_{p}-\chi_{f}\right)}{\left(\chi_{p}-\chi_{f}\right)+3} & H_{a}<\frac{\left(\chi_{p}-\chi_{f}\right)+3}{3 \chi_{p}} M_{s p} \\
M_{s p} / \mathrm{H}_{\alpha} & H_{a} \geq \frac{\left(\chi_{p}-\chi_{f}\right)+3}{3 \chi_{p}} M_{s p}\left(\left|\chi_{f}\right|<<1\right),
\end{array}\right.
$$

Where $\chi_{p}$ and $\chi_{f}$ are the magnetic susceptibilities of the particle and ambient fluid, respectively, $M_{\text {sn }}$ is saturation magnetization of the particle, and $H_{a}=\left|\mathbf{H}_{a}\right|$.

We assume nonmagnetic fluid $\left(\chi_{f} \approx 0\right)$ and high susceptibility of magnetic particles, i.e. $\chi_{p}>1$, which is in the case of water or air as fluid ambient, and magnetite $\left(\mathrm{Fe}_{3} \mathrm{O}_{4}\right)$ as particles accomplished; hence

$$
f(B)=\left\{\begin{array}{cl}
3 & B / \mu<M_{s p} / 3 \\
M_{s p} \mu / B & B / \mu \geq M_{s p} / 3
\end{array},\right.
$$

where $B$ is magnetic flux density of external field and is valid: $B / \mu=H_{a}$.

As an illustration we could calculate trajectory of magnetite particles in the magnetic field of permanent quadrupole. Magnetic flux density was modeled as magnetostatic problem by finite element method (FEM).

Magnetostatic problems are those in which the fields are time-independent. In this case, the field intensity $(\mathbf{H})$ and flux density (B) must obey equations

$$
\begin{gathered}
\nabla \times \mathbf{H}=\mathbf{J}, \\
\nabla \cdot \mathbf{B}=0,
\end{gathered}
$$

with a constitutive relationship between $B$ and $H$ for each material

$$
\mathbf{B}=\mu \mathbf{H} .
$$

If a material is nonlinear (e.g. saturating iron or alnico magnets), the permeability, $\mu$ is actually a function of $B$ :

$$
\mu=\frac{B}{H(B)} .
$$

Finite Element Method Magnetics (FEMM) goes about finding a field that satisfies all these 4 equations via a magnetic vector potential approach. Flux density is written in terms of the vector potential, $\mathbf{A}$, as:

$$
\mathbf{B}=\nabla \times \mathbf{A} .
$$

Then the first equation can be rewritten as:

$$
\nabla \times\left(\frac{1}{\mu(\mathbf{B})} \nabla \times \mathbf{A}\right)=\mathbf{J} .
$$

For a linear isotropic material ( $\mu=$ const. ) will previous expression reduce in Coulomb gauge $(\nabla \cdot \mathbf{A}=0)$ to

$$
-\nabla \cdot\left(\frac{1}{\mu} \nabla\right) \mathbf{A}=\mathbf{J},
$$

in that $\nabla \times(\nabla \times \mathbf{A})=\nabla(\nabla \cdot \mathbf{A})-\nabla \cdot \nabla \mathbf{A}$.

We consider solved problems as planar ones, flux density defined in $x-y$ plane, i.e. we regard $z$-coordinate of magnetic flux density, $B$, equals to zero, which means that magnetic vector potential is equal to $\mathbf{A}=(0,0, A)$. We also confine to current density parallel to $z$-direction of coordinate system. In this simplification last equation leads to scalar elliptic partial differential equation

$$
-\nabla \cdot\left(\frac{1}{\mu} \nabla A\right)=J
$$
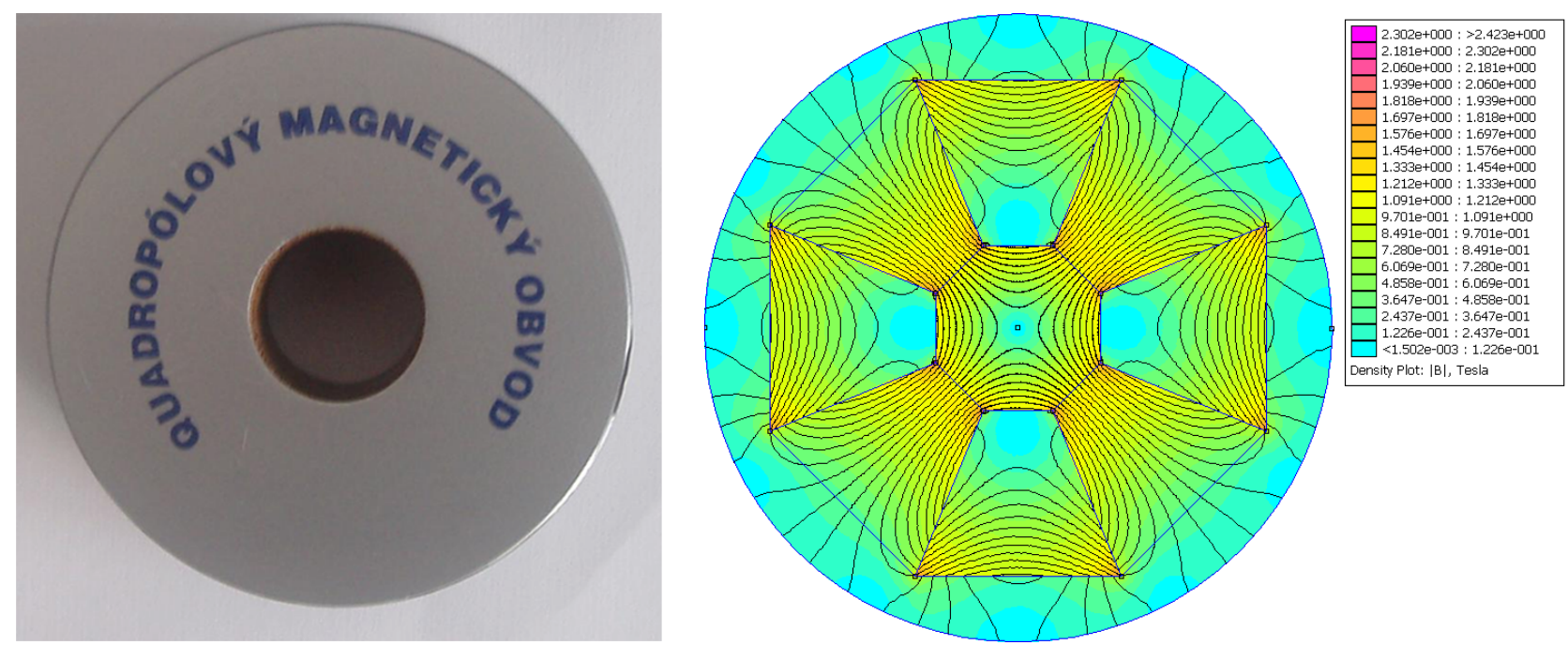

Fig. 3. Permanent quadrupole realized as an octapolar magnet, and its flux density obtained from FEMM model. 
the solution to which using finite elements method is the basis of the David Meeker FEMM ${ }^{31}$ program used in this study.

We have created a planar model of permanent quadrupole using this software, which is not $3 \mathrm{D}$, but it represents the distribution of flux density in transversal plane of infinite long permanent magnets arranged to quadrupole with zero value of perpendicular component of flux density. Our quadrupole consists of eight sphenoid blocks, in the section, of uniformly magnetized neodymium rareearth magnets with magnetic energy product $37 \mathrm{MG}$.Oe (megagauss-oersted; $\mathrm{NdFeB} \mathrm{N37)} \mathrm{and} \mathrm{with} \mathrm{magnetization}$ orientation revolved in $135^{\circ}$ between adjacent blocks. The geometry of quadrupole is determined by the radii of inscribed and circumscribed circle, i.e. 1.4 and $4.5 \mathrm{~cm}$ in our case, respectively. The maximal size of mesh elements in FEM model was $10^{-4} \mathrm{~m}$.

We have already built such a quadrupolar magnetic circuit (Fig. 3) for the puposes of magnetic targeting into lung, as illustrated on Fig. 4.

In our model we consider in addition to magnetic force fluidic force acting on a moving particle in fluid medium. Its magnitude is determined by Stokes' law for the drag on a sphere with radius $R_{p}$ in uniform flow,

$$
\mathbf{F}_{s}=-6 \pi \eta R_{p}\left(\mathbf{v}_{p}-\mathbf{v}_{f}\right)
$$

where $\eta$ and $\mathbf{v}_{f}$ are the viscosity and the velocity of the fluid, respectively, and $\mathbf{v}$ is the velocity of the particle. In our case is the fluid ambient quiescent, i.e. $v_{f}=0 \mathrm{~m} . \mathrm{s}^{-1}$.

In the next step computed magnetic flux density of permanent quadrupole by FEM analysis were extracted from FEMM and used for calculation of particles trajectory. Movement of magnetic particles in the plane in magnetic field with flux density $B$ in fluid ambient with viscosity $\eta$, which is not moving, is described by system of ordinary differential equations (ODE):

$$
\begin{aligned}
& \frac{\mathrm{d} x}{\mathrm{~d} t}=v_{p, x} \\
& \frac{\mathrm{d} y}{\mathrm{~d} t}=v_{p, y} \\
& \frac{\mathrm{d} v_{p, x}}{\mathrm{~d} t}=\frac{1}{m_{p}}\left\{\frac { V _ { p } } { \mu } f ( B ( x , y ) ) \left[+B_{x}(x, y) \frac{\partial B_{x}(x, y)}{\partial x}+\right.\right. \\
& \left.\left.+B_{y}(x, y) \frac{\partial B_{x}(x, y)}{\partial y}\right]-6 \pi \eta R_{p} v_{p, x}\right\} \\
& \frac{\mathrm{d} v_{p, y}}{\mathrm{~d} t}=\frac{1}{m_{p}}\left\{\frac { V _ { p } } { \mu } f ( B ( x , y ) ) \left[+B_{x}(x, y) \frac{\partial B_{y}(x, y)}{\partial x}+\right.\right. \\
& \left.\left.+B_{y}(x, y) \frac{\partial B_{y}(x, y)}{\partial y}\right]-6 \pi \eta R_{p} v_{p, y}\right\}
\end{aligned}
$$

(14). Gradient of flux density components occurred in mentioned expression was calculated numerically in each point of trajectory from the definition of derivation by replacing limit of space element by the element of dimension ten times smaller than maximal size of mesh elements of the FEM model.

For setting the trajectory of magnetite $\left(\mathrm{Fe}_{3} \mathrm{O}_{4}\right)$ particles, with density a $\rho_{p}=5000 \mathrm{~kg} \cdot \mathrm{m}^{-3}$ and a saturation magnetization $M_{s p}=4.78 \times 10^{5} \mathrm{~A} \cdot \mathrm{m}^{-1}$, in the magnetic field created by quadrupole consisting of permanent NdFeB N37 magnets, which is represented by magnetic flux density lines and flux density $B$ in Fig. 3., in nonmoving and nonmagnetic fluid, with viscosity equal to that of water or air, i.e. $\eta=1.003 \times 10^{-3}$ N.s.m ${ }^{-2}$ or $\eta=1.82 \times 10^{-5}$ N.s.m ${ }^{-2}$, respectively, when we have considered the theoretical model described in previous section, by solving ODE system (7), we have used ode 23s numerical solver of software Matlab 7.0 (The MathWorks, 2004). Computations were done for nanoparticles with radius $50 \mathrm{~nm}$, which are often used in magnetic drug targeting, as well as microparticles with radius $10 \mu \mathrm{m}$, which can be used for magnetic separation.

There are shown snapshots of trajectories of hundred magnetite particles of each size in the air as fluid ambient

where $m_{p}=V_{p} \rho_{p}$ and $V_{p}=4 / 3 \pi R_{p}^{3}$ are the mass and the volume of particle, respectively. This system was obtained by combination of expressions (1)-(3), (5) and 
in different times in Fig. 5, where $t_{\max }$ is the capture time of the slowest particle. Initial conditions for calculations were: randomly position in the circle with radius $0.4 \mathrm{~cm}$ from the center of quadrupole and zero initial velocity. Computation of trajectory for each particle was stopped after it reached internal boundary of quadrupole, approximately at a distance $1.4 \mathrm{~cm}$ from the center. As we can see microparticles were captured very fast in about $3 \mathrm{~ms}$, also small nanoparticles were attracted in a longer, but still reasonable time approximately after $16 \mathrm{~s}$. There were shown $^{32}$ similar capture times for motion of magnetite particles of same sizes in the analytically described mag- netic field of ideal high-current wire quadrupole driven by current in about $5000 \mathrm{~A}$.

Long movement time can be diminished using the magnetic particles of greater size. We have made calculations for air as fluid medium, because aerosols of magnetic particles have the potential in targeted drug delivery by magnetic field to the lungs ${ }^{33,34}$. Mean capture time is in this case shorter than ones in viscously water medium, due to smaller drag force. Capture time of magnetic particles in magnetic field of quadrupole, can be also decreased by increasing magnetic flux densities and field gradients of studied configuration replacing
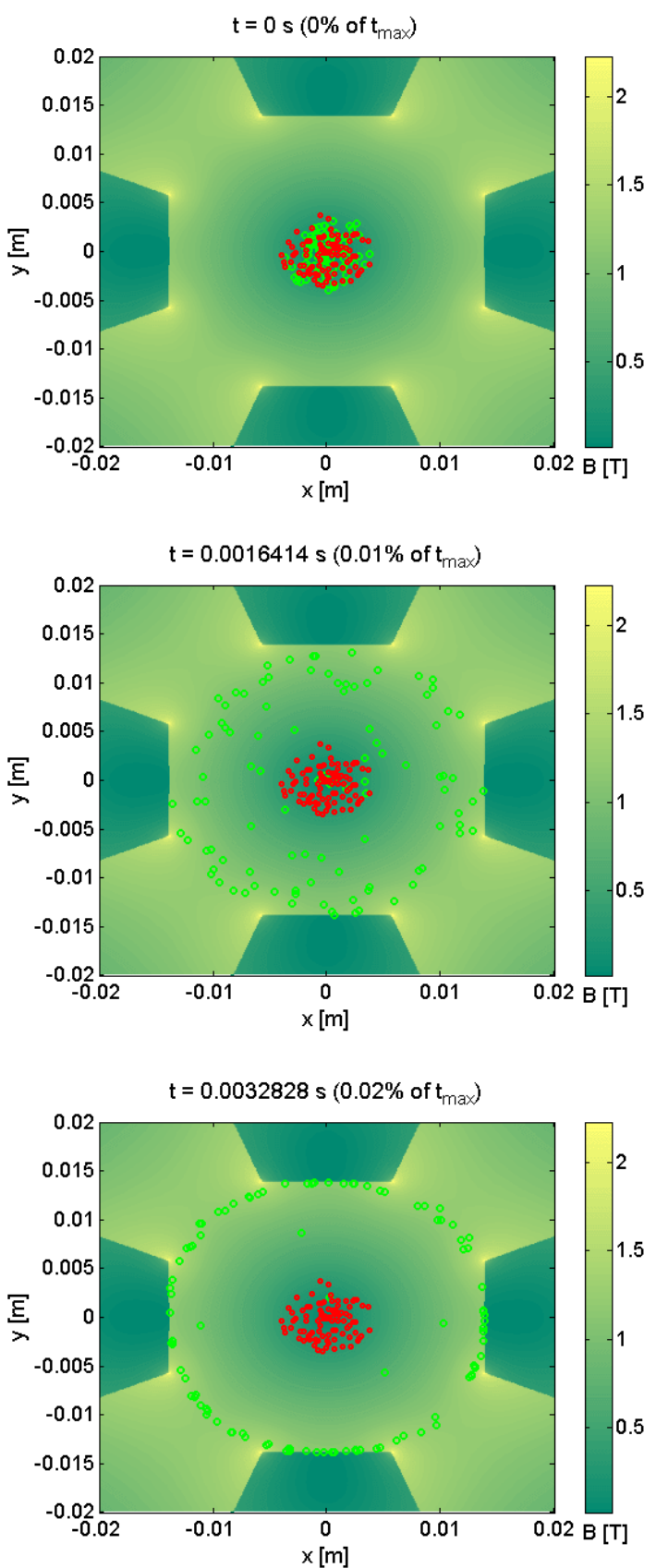
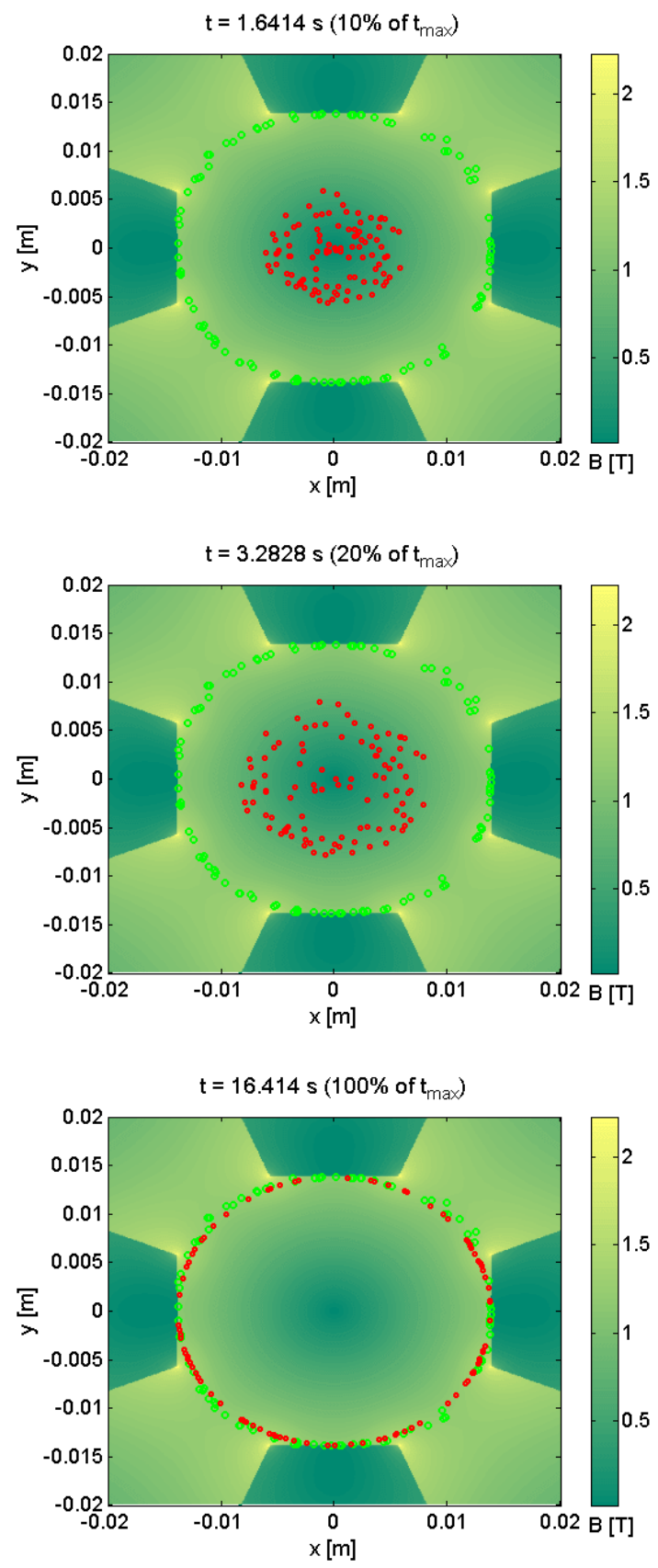

Fig. 5. Snapshots of trajectories of magnetite particles with different sizes, microparticles (green) with radius $10 \mu \mathrm{m}$ and nanoparticles (red) with radius $50 \mathrm{~nm}$, in the field of permanent quadrupole in the air, in different times, where $t_{\max }$ is time of the movement of the slowest particle. 
permanent magnets by pulsed electromagnets, similar to those pulsed quadrupole lenses using for focusing heavy ion beams in accelerator physics. These results equally apply also to magnetic separation of proteins, DNA, and whole cells ${ }^{35,36}$.

The first study about the magnetic drug targeting was performed 30 years ago ${ }^{37}$, but the revival of the field, due to apperance of a cheap and very powerful neodymium magnets, started in the the new millenium ${ }^{38-44}$. These principles are used also in magnetofection (MF) which is a method in which magnetic nanoparticles associated with vector DNA are transfected into cells by the influence of an external magnetic field. For this purpose, magnetic particles might be coated with the polycation polyethylenimine. These complexes readily associate with negatively charged DNA since the magnetic particles are positively charged due to the polyethylenimine. Whether viral or nonviral vectors, MF has been shown to enhance the efficiency of the vectors up to several thousand times ${ }^{45,46}$. Also combination of magnetic targeting with controlled release seems to be a very promising field ${ }^{47-51}$. Moreover magnetic nanoparticles provide magnetic resonance contrast enhancement (i.e., changes in signal intensity) by shortening both the longitudinal and transverse relaxation of surrounding protons, and widely available MRI equipment can be used for their noninvasive tracking in the organism ${ }^{52-54}$.

\section{CONCLUSIONS}

Functional magnetic nanoparticles offer improved spatio-temporal control over drug kinetics and distribution, thus opening the prospect of safer and more specific therapies. We found an explosive growth of published papers from this field of nanomedicine, showing the almost unlimited potential of magnetic nanoparticles in the field of experimental and clinical oncology.

\section{ACKNOWLEDGEMENTS}

This work was supported by VEGA No.: grant 1/0082/08 and Magseletofection project of 6. FP of EU under the contract No.: LSHB-CT-2006-019038.

\section{REFERENCES}

1. Laufberger V. Sur la cristallisation de la ferritine. Bull Soc Chim Biol 1937;19:1575.

2. Kohgo Y, Ikuta K, Ohtake T, Torimoto Y, Kato J. Body iron metabolism and pathophysiology of iron overload. Int J Hematol 2008 ;88(1):7-15

3. Pankhurst Q, Hautot D, Khan N, Dobson J. Increased levels of magnetic iron compounds in Alzheimer's disease. J Alzheimer's Dis $2008 ; 13(1): 49-52$.

4. Babincová M, Babinec P. Dopamine mediated iron release from ferritin is enhanced at higher temperatures: Possible implications for fever-induced Parkinson's disease. J Magn Magn Mater 2005;293(1):341-4.
5. Kronick P, Gilpin RW. Use of superparamagnetic particles for isolation of cells. J Biochem Biophys Methods 1986;12(1-2):73-80.

6. Simsek E, Akif Kilic M. Magic ferritin: A novel chemotherapeutic encapsulation bullet. J Magn Magn Mater 2005;293(1):509-13.

7. Gilad AA, Winnard Jr. PT, van Zijl PCM, Bulte JWM. Developing MR reporter genes: Promises and pitfalls. NMR Biomed 2007;20(3):275-90.

8. Babincová M, Leszczynska D, Sourivong P, Babinec P. Selective treatment of neoplastic cells using ferritin-mediated electromagnetic hyperthermia. Med Hypotheses 2000;54(2):177-9.

9. Lee S, Lee H, Park J, Choi H, Han K, Seo H, et al. A novel approach to ultrasensitive diagnosis using supramolecular protein nanoparticles. FASEB Journal 2007;21(7):1324-34.

10. Gider S, Awschalom DD, Douglas T, Mann S, Chaparala M. Classical and quantum magnetic phenomena in natural and artificial ferritin proteins. Science 1995; 268(5207):77-80.

11. Morrish AH. Physical Principles of Magnetism. New York, IEEE Press;2001.

12. Jiles D. Introduction to Magnetism and Magnetic Materials, London, Chapman and Hall; 1991.

13. Schwertmann U, Cornell RM. Iron oxides in the laboratory: preparation and characterization. Weinheim, VCH;1991.

14. Gupta AK, Gupta M. Synthesis and surface engineering of iron oxide nanoparticles for biomedical applications. Biomaterials 2005;26(18):3995-4021.

15. Berry CC, Curtis ASG. Functionalisation of magnetic nanoparticles for applications in biomedicine. J Phys D 2003;36(13):R198206.

16. Sajja HK, East MP, Mao H, Wang YA, Nie S, Yang L. Development of multifunctional nanoparticles for targeted drug delivery and noninvasive imaging of therapeutic effect. Current Drug Discovery Technologies 2009;6(1):43-51.

17. Kikumori T, Kobayashi T, Sawaki M, Imai T. Anti-cancer effect of hyperthermia on breast cancer by magnetite nanoparticleloaded anti-HER2 immunoliposomes. Breast Cancer Res Treat 2009;113(3):435-41.

18. Veiseh O, Kievit FM, Gunn JW, Ratner BD, Zhang M. A ligandmediated nanovector for targeted gene delivery and transfection in cancer cells. Biomaterials 2009;30(4):649-57.

19. Kim D, Kim K, Kim K, Lee Y. Targeting to carcinoma cells with chitosan- and starch-coated magnetic nanoparticles for magnetic hyperthermia. Journal of Biomedical Materials Research - Part A 2009;88(1):1-11.

20. Cheng C, Chu P, Chuang K, Roffler SR, Kao C, Tseng W. Hapten-derivatized nanoparticle targeting and imaging of gene expression by multimodality imaging systems. Cancer Gene Ther 2009;16(1):83-90.

21. Mohapatra S, Mallick SK, Maiti TK, Ghosh SK, Pramanik P. Synthesis of highly stable folic acid conjugated magnetite nanoparticles for targeting cancer cells. Nanotechnology 2007;18(38).

22. Babincová M. Microwave induced leakage of magnetoliposomes. possible clinical implications. Bioelectrochem Bioenerget 1993;32(2):187-9.

23. Babincová M. Simple preparation and separation of magnetoliposomes. Chem Listy 1998;92(4):323.

24. Babincová M, Machová E. Magnetoliposomes may be useful for elimination of HIV from infected individuals. Zeitschrift fur Naturforschung - Section C Journal of Biosciences 1998;53(910):935-6.

25. Babincová M, Babinec P. Controlled drug delivery using magnetoliposomes. Cellular and Molecular Biology Letters 1997;2(1):3-7.

26. Babincova M, Babinec P. Possibility of magnetic targeting of drugs using magnetoliposomes. Pharmazie 1995;50(12):828-9.

27. Ferrari M. Cancer nanotechnology: Opportunities and challenges. Nature Reviews Cancer 2005;5(3):161-71.

28. Babincová M, Babinec P, Bergemann C. High-gradient magnetic capture of ferrofluids: Implications for drug targeting and tumor embolization. Zeitschrift fur Naturforschung - Section C Journal of Biosciences 2001;56(9-10):909-11.

29. Pankhurst QA, Connolly J, Jones SK, Dobson J. Applications of magnetic nanoparticles in biomedicine. J Phys D 2003;36(13):R167-81. 
30. Furlani EP, Sahoo Y. Analytical model for the magnetic field and force in a magnetophoretic microsystem. J Phys D 2006;39(9):1724-32.

31. Meeker D. software: Finite Elements Methods Magnetics, V 4.2. Available from: http://femm.foster-miller.net

32. Krafčík A, Babincová M, Babinec P. Theoretical analysis of mag netic particle trajectory in high-current pulsed quadrupole: implications for magnetic cell separation, drug targeting, and gene therapy Optoel. Adv. Mater. - Rapid Commun 2009;3(11): 226-231.

33. Babincová M, Babinec P. Aerosolized VEGF in combination with intravenous magnetically targeted delivery of DNA-nanoparticle complex may increase efficiency of cystic fibrosis gene therapy. Med Hypotheses 2006;67(4):1002.

34. Dames P, Gleich B, Flemmer A, Hajek K, Seidl N, Wiekhorst F, et al. Targeted delivery of magnetic aerosol droplets to the lung. Nature Nanotechnology 2007;2(8):495-9.

35. Šafařík I, Šafaříková M. Use of magnetic techniques for the isolation of cells. Journal of Chromatography B: Biomedical Sciences and Applications 1999;722(1-2):33-53

36. Šafaŕík I, Šafaříková M. Magnetic nanoparticles and biosciences Monatshefte fur Chemie 2002;133(6):737-59.

37. Widder KJ, Senyei AE, Ranney DF. In vitro release of biologically active adriamycin by magnetically responsive albumin microspheres. Cancer Res 1980;40(10):3512-7.

38. Gupta PK, Hung CT. Magnetically controlled targeted microcarrier systems. Life Sci 1989;44:175-86.

39. Lübbe AS, Bergemann C, Riess H, Schriever F, Reichardt P, Possinger K, et al. Clinical experiences with magnetic drug targeting: A phase I study with 4'-epidoxorubicin in 14 patients with advanced solid tumors. Cancer Res 1996;56(20):4686-93.

40. Lübbe AS, Bergemann C, Brock J, McClure DG. Physiological aspects in magnetic drug-targeting. J Magn Magn Mater 1999;194(1):149-55.

41. Babincová M, Altanerová V, Lampert M, Altaner C, Machová E, Šrámka M, et al. Site-specific in vivo targeting of magnetoliposomes using externally applied magnetic field. Zeitschrift fur Naturforschung - Section C Journal of Biosciences 2000;55(34):278-81.

42. Babincová M, Leszczynska D, Sourivong P, Babinec P, Leszczynsk J. Principles of magnetodynamic chemotherapy. Med Hypotheses 2004;62(3):375-80.
43. Babincova M, Altanerova V, Altaner C, Bergemann C, Babinec P. In vitro analysis of cisplatin functionalized magnetic nanoparticles in combined cancer chemotherapy and electromagnetic hyperthermia. IEEE Transactions on Nanobioscience 2008;7(1):15-9.

44. Misra RDK. Magnetic nanoparticle carrier for targeted drug delivery: Perspective, outlook and design. Materials Science and Technology 2008;24(9):1011-9.

45. Scherer F, Anton M, Schillinger U, Henke J, Bergemann C, Kruger A, Gansbacher B, Plank C. Magnetofection: enhancing and targeting gene delivery by magnetic force in vitro and in vivo. Gene Ther 2002;9:102-9.

46. Mykhaylyk O, Zelphati O, Hammerschmid E, Anton M, Rosenecker J, Plank C. Recent advances in magnetofection and its potential to deliver siRNAs in vitro. Methods Mol Biol 2009;487:111-46.

47. Babincová M, Leszczynska D, Sourivong P, Babinec P. Picosecond laser pulses mediated drug release from magnetoliposomes. Cellular and Molecular Biology Letters 1999;4(4):625-30.

48. Babincová M, Leszczynska D, Sourivong P, Čičmanec P, Babinec P. Superparamagnetic gel as a novel material for electromagnetically induced hyperthermia. J Magn Magn Mater 2001;225(1-2):10912.

49. Babincová M, Čičmanec P, Altanerová V, Altaner C, Babinec P. AC-magnetic field controlled drug release from magnetoliposomes: Design of a method for site-specific chemotherapy. Bioelectrochemistry 2002;55(1-2):17-9.

50. Babincová M, Altanerová V, Altaner C, Čičmanec P, Babinec P. In vivo heating of magnetic nanoparticles in alternating magnetic field. Med Phys 2004;31(8):2219-21.

51. Babinec P, Babincová M, Sourivong P, Leszczynska D. Efficient treatment of pigmented B16 melanoma using photosensitized long-circulating magnetofullerenosomes. J Magn Magn Mater 2005;293(1):394-7.

52. Chouly C, Pouliquen D, Lucet I, Jeune JJ, Jallet P. Development of superparamagnetic nanoparticles for MRI: Effect of particle size, charge and surface nature on biodistribution. J Microencapsul 1996;13(3):245-55.

53. Babinec P, Babincová M. Towards multimodal nanoparticle labels for molecular imaging of biological processes. Med Hypotheses 2007;69(3):703-4.

54. Syková E, Jendelová P, Herynek V. MR tracking of stem cells in living recipients. Methods Mol Biol 2009;549:197-215. 